\title{
Influencia del capital humano en la calidad de la auditoría contable
}

\author{
Mónica Fernández ${ }^{1}$, Ana Gisbert ${ }^{2}$, Juan Carlos Salazar ${ }^{2}$ \\ ${ }^{1}$ Universidad Europea, ${ }^{2}$ Universidad Autónoma (Spain) \\ monica.fernandez@uem.es, ana.gisbert@uam.es, juancarlos.salazar@uam.es
}

Received October, 2013

Accepted November, 2013

\section{Resumen}

Objeto: Se estudian los determinantes de calidad del capital humano y del servicio en empresas de auditoría y principalmente cómo cualidades del capital humano inciden en la calidad del servicio prestado.

Diseño/metodología: Se realizó un cuestionario a una muestra de socios de una firma de auditoría relevante. Con los datos de los cuestionarios se obtuvieron los mejores indicadores de calidad de capital humano y calidad del servicio respectivamente. Posteriormente, se elaboran tablas de contingencia, con los datos tratados, para determinar las probabilidades de encontrar equipos con elevado nivel de capital humano, equipos con elevado nivel de servicio y ver si dentro de los equipos con alto desarrollo de su personal, el nivel del servicio es más alto o no.

Aportaciones $\boldsymbol{y}$ resultados: La calidad del factor humano es un activo intangible determinante en las organizaciones, especialmente en las que prestan servicios que requieren una alta capacitación profesional como ocurre en el sector de auditoría. Los resultados ponen de manifiesto que determinadas capacidades del capital humano inciden de forma relevante en factores asociados a la calidad del servicio de auditoría. El análisis realizado sugiere que una buena gestión e inversión en el capital humano de las mencionadas empresas, incide en la calidad del servicio prestado al cliente.

Limitaciones: El trabajo se ha podido realizar en una única firma de auditoría y la muestra no ha sido muy elevada. Pretendemos conseguir ampliar las muestras y las firmas participantes en próximas investigaciones. 
Valor añadido: En este análisis la variable más destacada, en cuanto a capital humano se refiere, es la experiencia y formación en la industria. Políticas de buena gestión del capital humano pueden fomentar una cultura donde el objetivo de ética y transparencia, contribuyan a mejorar los servicios prestados.

Palabras clave: Activo intangible, Calidad del capital humano, Calidad del servicio de auditoría, Firma de auditoría

Códigos JEL: M100, M420

Title: Influence of human quality on financial statement audit quality

\section{Abstract}

Purpose: We studied the determinants of human capital quality and service in auditing companies and especially how human capital qualities affect the quality of the service.

Design/methodology/approach: We sent a questionnaire to a sample of members of a relevant audit firm. With the data were obtained the best indicators of human capital and service quality respectively. Subsequently, contingency tables are made, with processed data, to determine the likelihood of finding high equipment level of human capital, equipment with high level of service and see if within teams with high development of its staff, the level of service is higher or not.

Findings: Quality on human capital is a key intangible asset in organizations, particularly those centered in providing services that require high professional training and expertise, as the auditing sector. The results show that certain capabilities of human capital have a relevant influence on service quality. And suggest that an appropriate investment in human capital is crucial in audit quality.

Research limitations: The work has been done on a single auditor and the sample was not very large. We are working to extend samples and firms participating in future researches.

Originality/value: In this analysis, the variable most important in terms of human capital, is the experience and training in the industry. Policies of good human capital management can foster a culture where the goal of ethics and transparency, help to improve services. 
Keywords: Intangible asset, Human capital Quality, Audit quality, Audit firm.

Jel Codes: M100, M420

\section{Introducción}

En el marco de la teoría de la agencia, la separación entre la dirección (agente) y los accionistas de la empresa (principal), hace necesario establecer mecanismos que permitan garantizar un apropiado flujo de información y comunicación entre ambos. En este sentido la información económico-financiera es esencial no sólo para garantizar la toma de decisiones de inversión por parte del principal, sino también para favorecer el establecimiento de contratos que permitan aunar los intereses de las partes. Por ello según como manifiestan $M$. Jensen y Meckling (1976), garantizar la calidad de la información económico-financiera utilizada en la toma de decisiones y en la formalización de contratos entre el agente y el principal, es un requisito indispensable para garantizar el éxito de esta relación.

Uno de los principales mecanismos de control existentes para garantizar la calidad de la información económico-financiera es la auditoría contable, una actividad de servicios en la que el principal activo de la empresa que los suministra lo constituye la capacidad de su equipo de generar valor a su cliente. Por este motivo, entre las empresas de servicios profesionales y en concreto en el sector de auditoría, la inversión y una apropiada gestión de su capital humano debe ser determinante para mejorar la calidad del servicio ofrecido, el grado de satisfacción del cliente, y en definitiva, la calidad de la información económico-financiera suministrada al conjunto de agentes interesados (stakeholders) y así en última instancia determine el éxito de una organización (Cappelli \& Singh, 1992). Sin embargo, ¿podemos observar fácilmente esta relación?, es decir, ¿cómo inciden las características del capital humano de las empresas de auditoría en la calidad del servicio?

Por otro lado, el trabajo se sitúa en el marco de la teoría de recursos y capacidades que se fundamenta principalmente en la creación por parte de la empresa de capital humano (Delery \& Shaw, 2001) que se constituya en la base para la consecución de ventajas competitivas. Hemos de tener claro, en base la literatura existente sobre la mencionada teoría, que las políticas de recursos humanos influyen positivamente en los resultados empresariales. En este sentido, reconocemos que disponer del capital humano adecuado es una condición necesaria pero no suficiente para la obtención de resultados superiores, de tal forma que esta relación se encuentra supeditada a que la empresa consiga motivar a los trabajadores para que desarrollen ese capital humano, establecer un clima laboral favorable, altos niveles de satisfacción laboral y reducciones en los niveles de absentismo y rotación. El capital humano, 
puede ser definido como la capacidad colectiva de la organización para conseguir los resultados deseados mediante la utilización del conocimiento de los trabajadores, y puede ser considerado como la suma de la experiencia de los trabajadores, de sus conocimientos y de sus aptitudes (Tseng \& James, 2005). Además, el capital humano existente en una organización puede convertirse en fuente de ventajas competitivas mejorando los niveles de productividad, así como la satisfacción de los clientes (Youndt \& Snell, 2004).

El objetivo de este trabajo es contribuir a la literatura en materia de la calidad de auditoría, centrándose en la incidencia que el capital humano tiene directamente en el servicio de auditoría. A través del desarrollo de una encuesta a un conjunto de socios de una empresa multinacional de servicios de auditoría, los resultados ponen de manifiesto la relevancia de la inversión en el personal de las compañías de servicios profesionales, desarrollando no sólo sus conocimientos técnicos sino también determinadas habilidades relacionales y personales como elemento clave de mejora y diferenciación de la cartera de servicios que prestan a sus clientes.

\section{Capital humano: pilar en la generación de valor de empresas auditoras.}

El valor real de una compañía no se muestra de forma completa en sus estados financieros, ya que en ellos no están presentes otros recursos generadores de valor económico como el capital intelectual de la empresa y la relación entre sus distintos componentes.

Al referirse al capital intelectual de una empresa, la definición más comúnmente aceptada, pero no por ello la primera ya que otros autores lo definieron antes, es la recogida en las "Directrices para la gestión y difusión de información sobre intangibles" propuestas en (Cañibano, Sánchez, García \& Chaminade, 2002), donde se establece que el capital intelectual se divide en tres categorías claramente diferenciadas e inter-relacionadas:

- capital humano,

- el capital estructural y

- el capital relacional.

El Capital humano está integrado por el conocimiento que el empleado se lleva cuando abandona la empresa, e incluye los saberes, las capacidades, experiencias y habilidades de las personas que integran la organización. Mientras una parte de este conocimiento es exclusivo de los individuos otra parte puede ser genérica. Ejemplos son la capacidad para innovar, la creatividad, el saber hacer y la experiencia previa, la capacidad para trabajar en equipo, la flexibilidad del empleado, la capacidad de negociación, la motivación, la satisfacción, la capacidad para aprender, la lealtad, etc., así como su nivel educativo y la titulación académica. 
Hudson (1993) lo considera como la herencia genética de cada individuo, su educación, su experiencia, sus actitudes en la vida. Bajo esta definición no sólo se recogen las características de las personas sino también el entorno en el que se integran. Stewart (1997) lo considera como el conjunto de capacidades de los individuos que son fuente de innovación y renovación dentro de las compañías.

Por ello, debemos distinguir entre características innatas y aquellas que son aprendidas. Así, podemos categorizar el capital humano en atributos personales, es decir, personalidad y otros atributos psicológicos tales como la inteligencia que son no modificables en un largo plazo y en habilidades, es decir, competencias y habilidades relacionadas con el trabajo que son potencialmente modificables (Carson, Ranzijn, Winefield \& Marsden, 2004).

En la última década, se ha analizado al capital humano como determinante del aumento de valor de las compañías que genera el denominado "valor añadido". Bontis y Fitz-end (2002) califican al capital humano como "la palanca de los beneficios en la economía del conocimiento". Con anterioridad, Sullivan (1998) consideraba el conocimiento o la inteligencia colectiva de la gente dentro de una organización como el más grande de los activos intangibles. Y según palabras de Fitz-End (2000) "los directivos han aceptado que las personas y no el dinero, las máquinas o equipos son los diferenciadores críticos de las empresas".

En las empresas de auditoría, el capital humano lo componen el conjunto de auditores que, en el desarrollo de sus funciones, no sólo necesitan un conocimiento técnico específico sino también de una serie de habilidades innatas que les permitan desarrollar con éxito los distintos proyectos abordados.

McKnight y Wright (2011) se refieren a una serie de factores determinantes del buen desarrollo de la profesión de auditor relacionados con:

- los conocimientos técnicos,

- la experiencia en el sector y conocimiento del cliente y

- las capacidades y las aptitudes personales (Tabla 1 ).

McKnight y Wright (2011) sugieren que el grado de desarrollo de un auditor en su trabajo impacta en la calidad de los estados financieros. Así estos autores afirman que los auditores que consiguen mayores conocimientos técnicos y habilidades de interacción con los clientes, así como un mejor comportamiento y actitud profesional, se consideran auditores de mayor desarrollo profesional, capaces de proporcionar mejores servicios. 
Para el desarrollo profesional como auditor, es imprescindible el conocimiento técnico obtenido a lo largo de la trayectoria profesional. Sin embargo, Bonner y Lewis (1990) no otorgan tanta relevancia a los años de experiencia sino al proceso de adquisición del conocimiento técnicopráctico necesario, así como a las habilidades necesarias para desarrollar el trabajo de auditoría. Moroney y Carey (2011) demuestran que la experiencia en el sector industrial del cliente tiene más impacto en el desarrollo del auditor, que la experiencia ganada en el desarrollo de los procedimientos propios de auditoría. Por este motivo, desde los años 80, las empresas de auditoría se han especializado por sectores y han explotado como parte de su modelo de negocio esta especialización. Este es el caso de las Big-Four de las que se hablará más adelante.

\begin{tabular}{|c|c|c|}
\hline Conocimientos técnicos & $\begin{array}{l}\text { Experiencia en el sector y } \\
\text { conocimiento del cliente }\end{array}$ & $\begin{array}{c}\text { Actitudes y } \\
\text { comportamientos } \\
\text { profesionales }\end{array}$ \\
\hline $\begin{array}{l}\text { Habilidad para resolver } \\
\text { problemas }\end{array}$ & $\begin{array}{c}\text { Conciencia del negocio del } \\
\text { cliente }\end{array}$ & Expresión escrita \\
\hline $\begin{array}{c}\text { Conocimientos en } \\
\text { contabilidad }\end{array}$ & $\begin{array}{l}\text { Entender las preocupaciones } \\
\text { del cliente }\end{array}$ & Expresión oral \\
\hline Análisis técnico & $\begin{array}{l}\text { Recomendar y asesorar al } \\
\text { cliente }\end{array}$ & Respeto \\
\hline $\begin{array}{l}\text { Operar dentro del } \\
\text { presupuesto }\end{array}$ & $\begin{array}{c}\text { Cumplir plazos dados por el } \\
\text { cliente }\end{array}$ & Confianza en uno mismo \\
\hline $\begin{array}{c}\text { Alto número de horas } \\
\text { facturables }\end{array}$ & $\begin{array}{c}\text { Respetar horarios y tareas en } \\
\text { el cliente }\end{array}$ & Responsabilidad \\
\hline $\begin{array}{l}\text { Respetar los principios } \\
\text { contables }\end{array}$ & $\begin{array}{l}\text { Entender los procesos } \\
\text { contables del cliente }\end{array}$ & Buen juicio \\
\hline
\end{tabular}

Tabla 1. Factores determinantes de la calidad del auditor. Fuente: McKnight y Wright (2011)

Tan (1999) demuestra la relevancia de factores asociados a las habilidades personales en el desarrollo profesional de un auditor, especialmente relevante a partir de la categoría de senior. Junto a las habilidades personales, los conocimientos técnicos son esenciales para desarrollar un buen trabajo de auditoría (Abdolmohammadi \& Shanteau, 1992). La educación y el conocimiento de los procedimientos y normativa contable y de auditoría son un requisito esencial en esta profesión. En la mejora de los conocimientos técnicos contribuye también la formación, que según Bonner y Lewis (1990), es determinante en la evolución de la carrera profesional del auditor. McKnight y Wright (2011) también señala la necesidad de tener la capacidad técnica de preparar informes o de ser capaz de identificar errores o sesgos que pueden existir en los estados financieros. Estas capacidades se incrementan en la medida en la que el auditor avanza en su carrera profesional. Además, la especialización por sectores empresariales, facilita la identificación de errores y sesgos en la información financiera (Tubbs, 1992), permitiendo reconsiderar y mejorar la precisión y el alcance de los procedimientos de auditoría inicialmente planificados (Tubbs, 1992; K. Jensen \& Payne, 2005; Moroney \& Carey, 2011). Según Tan (1999) el conocimiento del negocio del cliente es un factor especialmente relevante en el caso de los gerentes y socios de auditoría. Esta experiencia y especialización, 
unida a los conocimientos técnicos de contabilidad y auditoría, favorece la calidad del trabajo. De hecho, todas las compañías de auditoría más importantes del panorama internacional (Craswell, Francis \& Taylor, 1995) destacan por la especialización de sus equipos de trabajo en determinados sectores industriales.

En el tercer bloque de capacidades, las relacionadas con la capacidad de relación del auditor se concretan en la capacidad de escuchar al cliente para identificar sus preocupaciones, la capacidad de demostrar que el auditor entiende sus necesidades, la capacidad de resolver dudas y comunicar buenas prácticas de elaboración de la información financiera. Tan (1999) destaca la capacidad de proyectar una imagen de confianza y profesionalidad, como un factor esencial entre los socios. En definitiva, la habilidad relacional permite aportar valor añadido al servicio, a través de la correcta comunicación con el cliente. Esta parte se distingue del capital relacional (componente del capital humano) ya que éste último recoge el conjunto de recursos ligados a las relaciones externas de la empresa con sus clientes, proveedores de bienes, servicios o capital o con sus socios de I+D. Comprende tanto las relaciones de la empresa con terceros (inversores, acreedores, clientes, proveedores, etc.), como las percepciones que estos tienen de la compañía. Ejemplos de esta categoría son la imagen, la lealtad y la satisfacción de los clientes, los pactos con los proveedores, el poder comercial, la capacidad de negociación con instituciones financieras, con reguladores, etc. En la investigación nos estamos centrando en unas habilidades concretas de las personas, que por sí mismas constituyen el capital humano de las compañías, para relacionarse con sus clientes.

Pero junto a las capacidades del profesional de auditoría, la literatura empírica se ha centrado principalmente en analizar la incidencia de determinadas cuestiones regulatorias que indirectamente salvaguardasen la independencia del auditor, requisito indispensable para el desarrollo objetivo de las distintas pruebas y procedimientos que forman parte del proceso de auditoría. Francis (2011) argumenta que para garantizar la calidad del servicio de auditoría, junto a la mejora de los procedimientos y las pruebas aplicadas en el trabajo, es necesaria la competencia e independencia de los profesionales involucrados en el equipo de auditoría, que garantice la fiabilidad de la información económico-financiera de la empresa. La duración del nombramiento del auditor y su rotación obligatoria, ha sido uno de los factores analizados en numerosos estudios, como mecanismo de aproximación al impacto que la independencia del auditor puede tener sobre la calidad del servicio de auditoría. Según la legislación vigente, la rotación del auditor tiene como objetivo preservar la independencia del auditor, sin embargo, los estudios empíricos no corroboran beneficio alguno asociado al cambio obligatorio en el auditor. Algunos autores (Johnson, Khurana \& Reynolds, 2002) ponen de manifiesto que los nombramientos cortos (entre 2 y 3 años) y los medios (entre 4 a 8 años) están asociados a una disminución de la calidad en los estados financieros, mientras en que los nombramientos 
largos (más de 8 años) no hay evidencia de que la calidad de la auditoría se vea afectada. No obstante, esta disminución de la calidad se refleja principalmente en empresas que no forman parte del grupo de las conocidas Big four, donde los niveles de calidad de sus auditorías son muy elevados. Estas Big four son las cuatro grandes empresas de auditoría a nivel nacional e internacional que determinan que el mercado de auditoría esté muy concentrado ya que copan más del $95 \%$ del mismo.

Junto a la rotación del auditor, otro de los grandes factores analizados por su posible incidencia en la independencia y en definitiva, en la calidad de la auditoría, es el desarrollo simultáneo de trabajos de consultoría por la misma empresa que desarrolla el trabajo de auditoría. El argumento esgrimido por los organismos reguladores, que inicialmente quisieron limitar la prestación de otro tipo de servicios, se centraban en que el desarrollo simultáneo de trabajos de consultoría y auditoría por la misma empresa, podía incidir en la independencia del auditor. Existe una amplia literatura empírica que analiza esta cuestión, y que parece sin embargo concluir que en ningún caso hay una pérdida de independencia y por lo tanto una menor calidad del servicio de auditoria (Lim \& Tan ,2008; Willoughby et al., 2012).

Junto a ellos, cuestiones como la especialización de los equipos de trabajo por sectores ya habían sido previamente identificadas en la categoría relacionada con las habilidades técnicas del auditor (Balsam, Krishnan \& Yang, 2003; Dunn \& Mayhew, 2004). En cuanto a la supervisión del trabajo, Tubbs (1992) afirma que la supervisión del trabajo de los superiores ayuda a reforzar el conocimiento de los errores y reenfocar el trabajo, incidiendo en un mejor resultado final del servicio prestado al cliente.

En definitiva, la calidad del servicio de auditoría no sólo puede verse afectada por las capacidades y cualidades del capital humano, sino también por un conjunto de factores regulatorios de la profesión de auditoría cuyo objetivo es salvaguardar la independencia del auditor frente al cliente. El objetivo de este trabajo es dar un paso más, centrándose en el análisis de las principales capacidades personales, técnicas y relacionales de los auditores, como factor determinante de la calidad del servicio de auditoría.

\section{Hipótesis de trabajo}

Los auditores tienen incentivos y oportunidades para mejorar determinados atributos relacionados con el desarrollo de su trabajo de auditoría y así mejorar el rendimiento general en el mismo.

En este sentido, se han dividido los atributos relacionados con el trabajo de un auditor en tres grandes categorías (Abdolmohammadi \& Shanteau, 1992; Tan 1999): Habilidades personales, conocimientos técnicos y habilidades de relación con el cliente. 
Mientras que los conocimientos técnicos son esenciales, las habilidades personales y de relación con el cliente son cada vez significativamente más importantes para el desarrollo de la carrera profesional de un auditor. Especialistas en selección de personal en los campus universitarios, usan entrevistas para identificar competencias relacionadas con las habilidades de comunicación, la capacidad de relación con el cliente y el potencial para ser un miembro productivo de un equipo de auditoría. Para un auditor experimentado, es esencial tener amplias habilidades de relación con el cliente (Brown \& Wright, 2008). Estudios previos ponen de manifiesto que los auditores con un mayor desarrollo profesional, son conscientes de la relevancia de los conocimientos técnicos, las habilidades personales y la relación con el cliente en su desarrollo profesional (McKnhight \& Wright, 2011).

La esencia del trabajo de un auditor es la obtención y el tratamiento de evidencia, que le permitirá elaborar unas conclusiones sobre su trabajo (Libby, 1993). Un auditor que, a lo largo de su trabajo, tiene motivación, es perceptivo, enfoca las distintas situaciones que se encuentra como no rutinarias, se inclina más al uso de procedimientos estándar de auditoría y así tendrá diferente nivel de evidencia válida para formar su juicio de auditoría (Bonner \& Lewis, 1990; Tan et al., 2002). Además, estos auditores suelen ser más escépticos y tienen más seguridad en sí mismos, por lo que no llevarán a cabo juicios sin tener la suficiente evidencia (Hurtt, 2010). En este sentido, se asocia la confianza en los procedimientos estándar de auditoría y la motivación y habilidad de resolver problemas, con un grado más alto de desarrollo en el trabajo. Por otro lado, un rasgo que puede afectar al trabajo está vinculado con tener una personalidad más o menos proactiva Los más proactivos son asociados con mayor esfuerzo cognitivo y de ellos se espera mayor nivel de desarrollo en las tareas (Judge \& Bono, 2011 o 2001), también tienen más motivación ( $\mathrm{Ng}$ et al., 2006), mejores resultados académicos (Kalechstein \& Nowicki, 1997) y más alta capacidad de liderazgo ( $\mathrm{Ng}$ et al., 2006). A la vez, están más orientados a resultados que los menos proactivos que consideran que los resultados obtenidos en un trabajo, son atribuibles a las decisiones y comportamientos de otros. Se ha demostrado por varios autores como Chen y Silverthorne (2008) y Hyatt y Prawitt (2001), que hay una asociación entre una personalidad proactiva y mayores niveles de desarrollo en el trabajo.

Si el capital intelectual es un recurso generador de ventajas competitivas para las empresas y contribuye al desarrollo de las mismas, y el capital humano forma parte del capital intelectual, se proponen tres hipótesis generales para este trabajo centradas en la idea de que aquellos auditores que alcancen mayor desarrollo personal y profesional en atributos como los conocimientos técnicos y las habilidades personales y de relación con el cliente, podrán conseguir mejores resultados en su trabajo. En este sentido: 
- H1 - La calidad del capital humano de las empresas de auditoría, determinado por unas concretas habilidades personales de los empleados que han realizado el servicio, favorece la calidad del servicio prestado.

- H2 - La calidad del capital humano de las empresas de auditoría, determinado por unos concretos conocimientos técnicos de los empleados que han realizado el servicio, favorece la calidad del servicio prestado

- H3 - La calidad del capital humano de las empresas de auditoría, determinado por unas concretas habilidades de relación con el cliente de los empleados en el servicio, favorece la calidad del servicio prestado.

En cada uno de los tres grandes grupos en los que hemos dividido el capital humano: habilidades personales, conocimientos técnicos y habilidades de relación con el cliente se han fijado un total de 24 habilidades/capacidades ( 8 por cada grupo). Además, para analizar la calidad del servicio de auditoría se proponen 8 distintos indicadores. Por lo tanto, cada una de estas hipótesis generales se desdoblará en hipótesis específicas que relacionan cada una de las 8 facetas de la calidad del servicio de auditoría con las 24 habilidades/capacidades asociadas con el capital humano del equipo de auditoría. La hipótesis específica quedaría de la siguiente forma: El desarrollo de la capacidad/habilidad $X$ de capital humano tiene un impacto positivo en la faceta $Y$ de la calidad del servicio de auditoría.

\section{Análisis empírico}

\section{Desarrollo de la encuesta}

Para el desarrollo del análisis empírico, se elaboró un cuestionario con una relación de factores asociados a la calidad del capital humano que se han fundamentado en la teoría científica relacionada, así como a la calidad del servicio de auditoría. Los factores del capital humano se detallan en la Tabla 2 y están clasificados en tres categorías:

- habilidades personales,

- conocimientos técnicos,

- habilidades de relación con el cliente. 


\begin{tabular}{|c|c|c|}
\hline Habilidades personales & Conocimientos técnicos & Relación con el cliente \\
\hline $\begin{array}{l}\text { Habilidad de resolver } \\
\text { problemas encontrados en un } \\
\text { encargo de auditoría } \\
\text { Capacidad de expresión oral } \\
\text { y escrita } \\
\text { Habilidad de manejar la } \\
\text { responsabilidad y la presión } \\
\text { en el trabajo } \\
\text { Habilidad de ser perceptivo } \\
\text { Compromiso y motivación en } \\
\text { el desarrollo de sus funciones } \\
\text { Capacidad de ser proactivo y } \\
\text { tomar decisiones } \\
\text { Capacidad de trabajo en } \\
\text { equipo } \\
\text { Capacidad de liderazgo y } \\
\text { seguridad en sí mismo }\end{array}$ & $\begin{array}{l}\text { Conocimientos de } \\
\text { contabilidad y auditoria } \\
\text { Posesión de habilidad técnica } \\
\text { para preparar informes de } \\
\text { auditoría } \\
\text { Conocimiento de los } \\
\text { diferentes tipos de errores o } \\
\text { sesgos que pueden existir en } \\
\text { los estados financieros } \\
\text { Conocimiento del negocio del } \\
\text { cliente } \\
\text { Experiencia en los } \\
\text { procedimientos o tareas } \\
\text { propias del trabajo } \\
\text { Nivel educativo: postgrado y } \\
\text { título de auditor } \\
\text { Conocimiento de los sistemas } \\
\text { de control interno de los } \\
\text { clientes } \\
\text { Experiencia y formación en la } \\
\text { industria }\end{array}$ & $\begin{array}{l}\text { Capacidad de escuchar } \\
\text { atentamente para identificar } \\
\text { y entender las } \\
\text { preocupaciones del cliente } \\
\text { Capacidad de convencer al } \\
\text { cliente que entiendes sus } \\
\text { problemas y necesidades } \\
\text { Capacidad de comunicar } \\
\text { "buenas prácticas" de } \\
\text { información financiera } \\
\text { Capacidad de resolver dudas } \\
\text { al cliente en situaciones } \\
\text { concretas } \\
\text { Proyectar una imagen de } \\
\text { confianza y profesionalidad } \\
\text { Capacidad de resolución de } \\
\text { conflictos } \\
\text { Transmitir confidencialidad y } \\
\text { prudencia en el desarrollo del } \\
\text { trabajo } \\
\text { Capacidad de adaptación a } \\
\text { las situaciones particulares } \\
\text { del cliente }\end{array}$ \\
\hline
\end{tabular}

Tabla 2. Detalle de los factores relacionados con la calidad del capital humano (McKnight \& Wright, 2001; Tan, 1999)

Puede inferirse que si el conjunto de estas capacidades personales, técnicas y relacionales, mejoran el desarrollo profesional del auditor, también se verá favorecida la calidad del servicio prestado, es decir, la calidad de la auditoría.

Junto al análisis de las categorías relacionadas con la calidad del capital humano, se analizan una serie de factores directamente relacionados con la calidad del servicio de auditoría. Estos factores han sido seleccionados tomando como referencia la literatura empírica especializada, así como a través de conversaciones directas con responsables de la firma de auditoría seleccionada (el caso de los cinco primeros).

Como se señala más adelante, el análisis empírico se realiza a través de un cuestionario suministrado a un conjunto de socios de una multinacional de auditoría. El cuestionario consta de tres partes. En la primera, el socio encuestado debía dar su opinión profesional sobre cada uno de los factores recogidos en las Tablas 2 y 3 , según el grado de importancia que le otorgara. En la segunda parte del cuestionario, el socio encuestado debía seleccionar a dos de sus equipos de trabajo y valorar cada uno de ellos, tomando como referencia cada uno de los factores recogidos en el cuestionario. En la última parte se pregunta al socio por cuestiones generales tales como edad, sexo, años de experiencia, años de auditoría en el mismo cliente. 


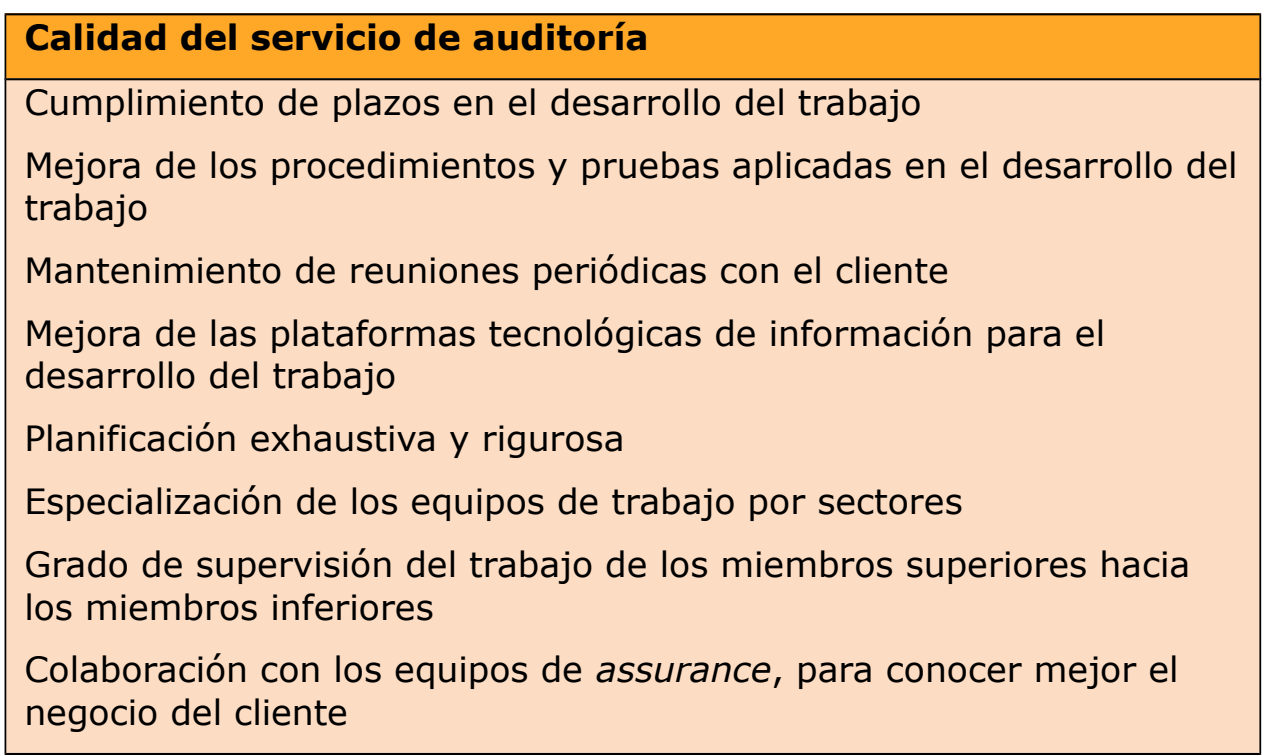

Tabla 3. Detalle de los factores que inciden en la calidad del servicio de auditoría

\section{Selección de los socios encuestados}

Para el desarrollo del análisis empírico se seleccionó una empresa del sector de auditoría con relevancia tanto en el ámbito nacional como internacional. Esta empresa seleccionada comparte una cultura organizativa donde el capital humano de la misma se rige por una serie de valores que citamos a continuación: liderazgo dando ejemplo, trabajo en equipo, respeto a la persona, análisis de los hechos para aportar un juicio profesional, comunicación de forma abierta y sincera, compromiso con la sociedad, actuación con integridad basándose en la ética.

Una vez seleccionada la firma, se identificó a un conjunto de socios en base a una serie de criterios de selección relacionados con:

- las características de sus clientes,

- el perfil personal y

- el perfil profesional.

De esta forma, los socios seleccionados estaban directamente relacionados con equipos de trabajo asociados a clientes de distintas características en cuanto a tamaño, cotización bursátil, facturación o antigüedad respecto a su relación con la firma de auditoría. Asimismo, se seleccionó a socios con distintos niveles de desarrollo de su carrera profesional, especializados en distintos sectores, hombres y mujeres de distintas franjas de edad. El cuestionario se puso a disposición de 25 socios durante el año 2012, siendo la tasa de respuesta del $61.53 \%$. En la tabla 4 se recogen las características de los 13 socios que finalmente respondieron. Como puede observarse, el conjunto de socios encuestados son responsables de la auditoría de empresas de distintos tamaños y tanto de sociedades cotizadas como no cotizadas. La 
selección exclusivamente de socios de auditoría, y no de auditores de otras categorías, se debe a que ellos son los responsables últimos de sus equipos de trabajo y del servicio que presta éste al cliente.

\begin{tabular}{|l|l|l|l|l|}
\hline $\begin{array}{c}\text { No } \\
\text { encuestas }\end{array}$ & Cotizada & \multicolumn{1}{c|}{$\begin{array}{c}\text { Nueva } \\
\text { auditoría }\end{array}$} & \multicolumn{1}{|c|}{ Facturación } & \multicolumn{1}{c|}{ Tamaño } \\
\hline 13 & NO: 8 socios & NO: 11 socios & $\begin{array}{l}\text { Más de 50 millones: 7 } \\
\text { socios }\end{array}$ & Grande: 7 socios \\
Sí: 5 socios & SÍ : 2 socios & $\begin{array}{l}\text { Inferior a 50 millones y } \\
\text { superior a 9: 3 socios } \\
\text { Inferior a 9 millones: } 3 \\
\text { socios }\end{array}$ & Pequeño: 3 socios \\
\hline
\end{tabular}

Panel A. Descripción de los socios encuestados en base a las características de sus clientes

\begin{tabular}{|l|c|c|}
\hline \multicolumn{2}{|c|}{ Criterio de selección } & No \\
\hline \multirow{2}{*}{ Edad } & $36-45$ años & 4 \\
\cline { 2 - 3 } & $46-55$ años & 4 \\
\hline \multirow{3}{*}{ Sector } & Masculino & 6 \\
\cline { 2 - 3 } & Femenino & 2 \\
\hline \multirow{2}{*}{ Experiencia } & CIM & 7 \\
\cline { 2 - 3 } & IGH & 1 \\
\hline Cliente & $8-10$ años & 7 \\
\hline & $0-3$ & 1 \\
\hline
\end{tabular}

$\mathrm{CIM}=$ Consumo, industrial y Telecomunicaciones; IGH = Infraestructuras, sector público y sanidad

Panel B. Descripción de la muestra según características personales y profesionales

Tabla 4. Características de los socios encuestados

\section{Análisis de resultados}

\section{Análisis descriptivo de la valoración realizada por los socios}

Un primer análisis descriptivo de los resultados obtenidos de la encuesta, ponen de manifiesto los factores que, en opinión de los socios, son determinantes de la calidad del capital humano, así como del propio servicio de auditoría. La Tabla 5 detalla el conjunto de factores de capital humano y de calidad de auditoría recogidos en la encuesta, que han sido valorados como "muy relevantes" por los socios encuestados. 


\begin{tabular}{|c|c|c|}
\hline Variable & Factor & $\begin{array}{l}\text { Tasa de respuesta } \\
\text { obtenida }\end{array}$ \\
\hline \multirow{6}{*}{ Capital humano } & $\begin{array}{l}\text { Proyectar una imagen de confianza y } \\
\text { profesionalidad }\end{array}$ & $100 \%$ \\
\hline & $\begin{array}{c}\text { Compromiso y motivación en el } \\
\text { desarrollo de las funciones }\end{array}$ & $93.75 \%$ \\
\hline & Nivel educativo & $93.75 \%$ \\
\hline & $\begin{array}{c}\text { Transmitir confianza y prudencia en el } \\
\text { desarrollo del trabajo }\end{array}$ & $87.50 \%$ \\
\hline & $\begin{array}{c}\text { Conocimientos de contabilidad y } \\
\text { auditoría }\end{array}$ & $75.00 \%$ \\
\hline & Capacidad de trabajo en equipo & $75.00 \%$ \\
\hline \multirow{2}{*}{ Calidad de auditoría } & $\begin{array}{l}\text { Cumplimiento de plazos en el } \\
\text { desarrollo del trabajo }\end{array}$ & $75.00 \%$ \\
\hline & $\begin{array}{l}\text { Mantenimiento de reuniones periódicas } \\
\text { con el cliente }\end{array}$ & $62.50 \%$ \\
\hline
\end{tabular}

Tabla 5. Mejores indicadores de capital humano y calidad de auditoría

Análisis a través de tablas de contingencia

Con el objetivo de valorar el impacto de las variables de capital humano en las variables de calidad de la auditoría, se realizó un análisis a través de tablas de contingencia con la puntuación obtenida por cada uno de los equipos valorados en la segunda parte de la encuesta. En el análisis a través de tablas de contingencia, Y se refiere a la variable "calidad del servicio de auditoría" y $X$ al conjunto de factores relacionados con la calidad del capital humano, de cada una de las tres dimensiones analizadas: habilidades personales (HP), conocimientos técnicos (CT) y relación con el cliente (RC).

Las tablas 6 a 8 recogen los principales resultados del análisis de contingencia realizado. En cada tabla, la columna $\mathrm{P}(\mathrm{Y})$ indica el porcentaje de equipos de trabajo que han sido muy bien valorados en relación a cada uno de los factores de calidad del servicio de auditoría analizados. Por otra parte, la columna $\mathrm{P}(\mathrm{Y} / \mathrm{X})$ hace referencia al porcentaje de equipos de trabajo con una puntuación elevada en los factores de calidad del servicio de auditoría, cuando de forma simultánea, obtienen una puntuación elevada en el conjunto de factores de capital humano analizados. La diferencia entre $P(Y / X)$ y $P(Y)$ permite inferir la existencia de una relación significativa (positiva o negativa) entre ambas, puesto que refleja la forma en la que el conjunto de factores de capital humano analizados inciden en la probabilidad de que el equipo obtenga una buena calificación en relación al servicio de auditoría. En definitiva las tablas analizan la incidencia de los distintos factores del capital humano sobre los factores de calidad del servicio de auditoría

Se descarta la variable "Colaboración con los equipos de assurance, para conocer mejor el negocio del cliente", al obtenerne que no existe vinculación con las variables independientes (de capital humano) debido a que no se da por encima de la media en ninguno de los equipos valorados. Según Lim y Tan (2008) existen estudios que muestran que la prestación de 
servicios de no auditoría en apariencia perjudican la independencia que debe regir el trabajo de un auditores pero también se ve que no existe este efecto o es muy débil (Willoughby et al., 2012) por lo que los resultados son contradictorios en relación a esta variable.

El "cumplimiento de plazos" había sido uno de los factores más valorados por el conjunto de socios encuestados. Como se recoge en la Tabla 6, el $75 \%$ de los socios encuestados, lo considera uno de los factores más relevantes asociados a la calidad del servicio de auditoría. Tal y como puede observarse en la Tabla 7, el 75\% de los equipos analizados, obtienen una buena valoración en cuanto al cumplimiento de plazos. Sin embargo, este porcentaje aumenta al $99,75 \%$ entre el conjunto de equipos de trabajo que de forma simultánea, obtienen una buena valoración respecto a la:

- capacidad de expresión oral y escrita,

- la experiencia y formación en la industria y

- la capacidad de comunicar "buenas prácticas" por lo que observamos que existe una relación positiva entre las variables de capital humano y la de servicio de auditoría cumplimiento de plazos.

\begin{tabular}{|c|c|c|c|c|}
\hline Tema & $\begin{array}{c}\text { Intangible (variable } \\
\text { independiente) }\end{array}$ & $\mathbf{P}(\mathbf{Y})$ & $\mathbf{P}(\mathbf{Y} / \mathbf{X})$ & $\begin{array}{c}\text { Variación de proporciones } \\
(\mathbf{P}(\mathbf{Y} / \mathbf{X})-\mathbf{P}(\mathbf{Y})\end{array}$ \\
\hline $\mathbf{C T}$ & $\begin{array}{c}\text { Experiencia y formación en } \\
\text { la industria }\end{array}$ & 75.00 & 99.75 & 24.75 \\
\hline $\mathbf{H P}$ & $\begin{array}{c}\text { Capacidad de expresión oral } \\
\text { y escrita }\end{array}$ & 75.00 & 99.75 & 24.75 \\
\hline $\mathbf{R C}$ & $\begin{array}{c}\text { Capacidad de comunicar } \\
\text { "buenas prácticas" de } \\
\text { información financiera }\end{array}$ & 75.00 & 99.75 & 24.75 \\
\hline
\end{tabular}

Tabla 6. Análisis de las variables de capital humano que indicen de forma más relevante en el "Cumplimiento de plazos"

Junto al cumplimiento de plazos, el "mantenimiento de reuniones periódicas con el cliente" ha sido uno de los factores que ha obtenido una mayor valoración por parte de los socios encuestados. Tal y como se pone de manifiesto en la tabla siguiente, la proporción de equipos en los que la valoración del mantenimiento de reuniones con el cliente es muy buena, asciende al $62,5 \%$ de total de equipos analizados. Sin embargo, este porcentaje aumenta significativamente, entre los equipos que poseen una elevada experiencia y formación en la industria, unos buenos conocimientos de contabilidad y auditoría y sobre todo, una alta habilidad de resolver problemas, donde vemos que aumenta hasta el $100 \%$. Podemos volver a apreciar la relación positiva anteriormente mencionada. 


\begin{tabular}{|c|c|c|c|c|}
\hline Tema & $\begin{array}{c}\text { Intangible (variable } \\
\text { independiente) }\end{array}$ & $\mathbf{P}(\mathbf{Y})$ & $\mathbf{P}(\mathbf{Y} / \mathbf{X})$ & $\begin{array}{c}\text { Variación de proporciones } \\
(\mathbf{P}(\mathbf{Y} / \mathbf{X})-\mathbf{P}(\mathbf{Y})\end{array}$ \\
\hline HP & $\begin{array}{c}\text { Habilidad de resolver } \\
\text { problemas encontrados en } \\
\text { un encargo de auditoría }\end{array}$ & 62.50 & 100.00 & 37.50 \\
\hline $\mathbf{C T}$ & $\begin{array}{c}\text { Conocimientos de } \\
\text { contabilidad y auditoría }\end{array}$ & 62.50 & 83.13 & 20.63 \\
\hline $\mathbf{C T}$ & $\begin{array}{c}\text { Experiencia y formación en } \\
\text { la industria }\end{array}$ & 62.50 & 80.00 & 17.50 \\
\hline
\end{tabular}

Tabla 7. Análisis de las variables de capital humano que indicen de forma más relevante en el

"Mantenimiento de reuniones periódicas con el cliente"

Este tipo de análisis se ha realizado sobre el conjunto de factores de calidad del servicio de auditoría analizados. La tabla 8 realiza una síntesis de resultados para el resto de los factores de calidad del servicio de auditoría analizados.

\begin{tabular}{|c|c|c|c|c|}
\hline Tema & $\begin{array}{l}\text { Intangible (variable } \\
\text { independiente) }\end{array}$ & $P(Y)$ & $\mathbf{P}(\mathbf{Y} / \mathbf{X})$ & $\begin{array}{l}\text { Variación de proporciones } \\
(\mathrm{P}(\mathrm{Y} / \mathrm{X})-\mathrm{P}(\mathrm{Y})\end{array}$ \\
\hline \multicolumn{5}{|c|}{$\begin{array}{l}\text { Panel A: "Mejora de los procedimientos y pruebas aplicados en el desarrollo del } \\
\text { trabajo" }\end{array}$} \\
\hline $\mathbf{R C}$ & $\begin{array}{l}\text { Capacidad de comunicar } \\
\text { "buenas prácticas" de } \\
\text { información financiera }\end{array}$ & 37.50 & 66.75 & 29.25 \\
\hline RC & $\begin{array}{l}\text { Capacidad de convencer al } \\
\text { cliente que entiendes sus } \\
\text { problemas y necesidades }\end{array}$ & 37.50 & 66.75 & 29.25 \\
\hline HP & Habilidad de ser perceptivo & 37.50 & 60.00 & 22.50 \\
\hline \multicolumn{5}{|c|}{ Panel B: Mejora de las plataformas tecnológicas } \\
\hline HP & $\begin{array}{l}\text { Capacidad de expresión oral } \\
\text { y escrita }\end{array}$ & 37.50 & 100.00 & 62.50 \\
\hline RC & $\begin{array}{l}\text { Capacidad de comunicar } \\
\text { "buenas prácticas" de } \\
\text { información financiera }\end{array}$ & 37.50 & 66.75 & 29.25 \\
\hline CT & $\begin{array}{c}\text { Conocimiento de los } \\
\text { sistemas de control interno } \\
\text { de los clientes }\end{array}$ & 37.50 & 55.50 & 18.00 \\
\hline
\end{tabular}




\begin{tabular}{|c|c|c|c|c|}
\hline Tema & $\begin{array}{c}\text { Intangible (variable } \\
\text { independiente) }\end{array}$ & $\mathbf{P}(\mathbf{Y})$ & $\mathbf{P}(\mathbf{Y} / \mathbf{X})$ & $\begin{array}{c}\text { Variación de proporciones } \\
(\mathbf{P}(\mathbf{Y} / \mathrm{X})-\mathrm{P}(\mathrm{Y})\end{array}$ \\
\hline \multicolumn{5}{|c|}{ Panel C: Planificación exhaustiva y rigurosa } \\
\hline $\mathbf{R C}$ & $\begin{array}{l}\text { Capacidad de convencer al } \\
\text { cliente que entiendes sus } \\
\text { problemas y necesidades }\end{array}$ & 25.00 & 44.50 & 19.50 \\
\hline CT & $\begin{array}{l}\text { Conocimiento de los } \\
\text { diferentes tipos de errores o } \\
\text { sesgos que pueden existir } \\
\text { en los estados financieros }\end{array}$ & 25.00 & 36.25 & 11.25 \\
\hline CT & $\begin{array}{c}\text { Conocimientos de } \\
\text { contabilidad y auditoría }\end{array}$ & 25.00 & 33.25 & 8.25 \\
\hline \multicolumn{5}{|c|}{ Panel D: Especialización de los equipos de trabajo por sectores } \\
\hline CT & $\begin{array}{c}\text { Experiencia y formación en } \\
\text { la industria }\end{array}$ & 43.80 & 80.15 & 36.35 \\
\hline CT & $\begin{array}{l}\text { Posesión de habilidad } \\
\text { técnica para preparar } \\
\text { informes de auditoría }\end{array}$ & 43.80 & 66.58 & 22.78 \\
\hline CT & $\begin{array}{c}\text { Conocimiento de los } \\
\text { sistemas de control interno } \\
\text { de los clientes }\end{array}$ & 43.80 & 55.63 & 11.83 \\
\hline \multicolumn{5}{|c|}{$\begin{array}{l}\text { Panel E: Grado de supervisión del trabajo de los miembros superiores hacia los } \\
\text { miembros inferiores }\end{array}$} \\
\hline HP & Habilidad de ser perceptivo & 43.80 & 80.15 & 36.35 \\
\hline RC & $\begin{array}{l}\text { Capacidad de resolución de } \\
\text { conflictos }\end{array}$ & 43.80 & 57.38 & 13.58 \\
\hline RC & $\begin{array}{c}\text { Capacidad de escuchar } \\
\text { atentamente para identificar } \\
\text { y atender las } \\
\text { preocupaciones del cliente }\end{array}$ & 43.80 & 54.75 & 10.95 \\
\hline
\end{tabular}

Tabla 8. Análisis de las variables de capital humano que indicen de forma más relevante en los factores de calidad del servicio de auditoría

Tal y como puede observarse en la Tabla 8 paneles A y B, el $37 \%$ de los equipos analizados, obtienen una buena valoración en cuanto a la mejora de los procedimientos y la mejora de las plataformas tecnológicas. Sin embargo, este porcentaje aumenta al $66,75 \%$ y $100 \%$, según la variable de calidad de servicio usada, entre el conjunto de equipos de trabajo que de forma simultánea, obtienen una buena valoración respecto a la capacidad de comunicar "buenas prácticas" y la capacidad de convencer al cliente que entiendes sus problemas así como la mejora de procedimientos y pruebas y por otro lado los que obtienen una buena valoración respecto a la capacidad de expresión oral y escrita, entre otras, y la mejora de las plataformas.

Por otro lado y tal como se manifiesta en el panel $C$, la proporción de equipos en los que la valoración de la planificación exhaustiva y rigurosa es muy buena, asciende al $25 \%$ de total de equipos analizados. Sin embargo, este porcentaje aumenta hasta el 44,5\%, entre los equipos que una tienen una elevada valoración en la planificación y una elevada valoración en la capacidad de convencer al cliente que entiendes sus problemas y necesidades. 
Finalmente, como se refleja en los paneles $D$ y $E$, la proporción de equipos en los que la valoración de la especialización y la supervisión es alta, asciende al 43,8\% de total de equipos analizados. Sin embargo, este porcentaje aumenta hasta el $80,15 \%$ entre el conjunto de equipos de trabajo que de forma simultánea, obtienen una buena valoración respecto a:

- la experiencia y formación en la industria así como en la especialización y

- habilidad de ser perceptivo así como en el grado de supervisión.

Del conjunto de los resultados recogidos en las tabla 6,7 y 8 cabe destacar que no todos los factores analizados parecen ser tan determinantes en la mejora de la calidad del proceso de auditoría. Del análisis de resultados, destacan 5 factores de carácter técnico, junto a 3 relacionados con habilidades personales y 4 de capacidades relacionales con los clientes. De estos factores, destaca la capacidad de comunicar "buenas prácticas de información financiera", que junto a la propia "experiencia y formación en la industria", índice en varios de los factores de calidad del servicio de auditoría analizados. Finalmente, "la capacidad de expresión oral y escrita" y la "habilidad de ser perceptivo" son las dos cualidades más relevantes del conjunto de habilidades personales analizadas tal y como observamos en la tabla 9.

\begin{tabular}{|l|l|l|}
\hline Habilidades personales & Conocimientos técnicos & Relación con el cliente \\
\hline $\begin{array}{l}\text { Capacidad de expresión oral y } \\
\text { escrita }\end{array}$ & $\begin{array}{l}\text { Experiencia y formación en la } \\
\text { industria }\end{array}$ & $\begin{array}{l}\text { Capacidad de comunicar } \\
\text { "buenas prácticas" de } \\
\text { información financiera }\end{array}$ \\
\hline Habilidad de ser perceptivo & $\begin{array}{l}\text { Conocimientos de } \\
\text { contabilidad y auditoria }\end{array}$ & $\begin{array}{l}\text { Capacidad de convencer al } \\
\text { cliente que entiendes sus } \\
\text { problemas y necesidades }\end{array}$ \\
\hline $\begin{array}{l}\text { Habilidad de resolver } \\
\text { problemas encontrados en un } \\
\text { encargo de auditoría }\end{array}$ & $\begin{array}{l}\text { Conocimiento de los sistemas } \\
\text { de control interno de los } \\
\text { clientes }\end{array}$ & $\begin{array}{l}\text { Capacidad de escuchar } \\
\text { atentamente para identificar y } \\
\text { entender las preocupaciones } \\
\text { del cliente }\end{array}$ \\
\hline \multirow{2}{*}{$\begin{array}{l}\text { Posesión de habilidad técnica } \\
\text { para preparar informes de } \\
\text { auditoría }\end{array}$} & $\begin{array}{l}\text { Capacidad de resolución de } \\
\text { conflictos }\end{array}$ \\
\cline { 2 - 3 } $\begin{array}{l}\text { Conocimiento de los } \\
\text { diferentes tipos de errores o } \\
\text { sesgos que pueden existir en } \\
\text { los estados financieros }\end{array}$ \\
\cline { 2 - 2 }
\end{tabular}

Tabla 9. Detalle de los factores relacionados con la calidad del capital humano, que inciden en la calidad del servicio de auditoría

\section{Conclusiones}

Este trabajo pone de manifiesto, en la empresa objeto de estudio, la existencia de determinados factores relacionados con las capacidades técnicas, relacionales y personales de los componentes de un equipo de auditoría, que pueden afectar de forma significativa a la 
calidad del servicio prestado a los clientes. El conocimiento de la industria, así como la capacidad de transmitir buenas prácticas de información financiera, son dos de los factores más relevantes, poniéndose de manifiesto cómo a través de la inversión en determinadas cualidades del capital humano en la empresa de auditoría analizada, se puede mejorar la calidad del servicio prestado y en última instancia, la calidad de la información económicofinanciera de las empresas. La mejora de ésta última ha sido estudiada por Lennox y Caramanis (2008); Dechow, Ge, Larson y Slolan (2011) desde otro punto de vista completamente distinto al nuestro.

Este trabajo muestra que la calidad de auditoría, no sólo se potencia a través de mecanismos regulatorios que limiten la actividad del sector, obligando a la rotación del auditor o limitando la participación en trabajos de consultoría. Como ha puesto de manifiesto la evidencia empírica en estos temas, los resultados no apoyan los argumentos a favor de la rotación obligatoria o la prohibición de colaborar en trabajos de consultoría. Sin embargo, el resultado de este trabajo nos revela que políticas adecuadas de gestión del capital humano en la empresas de auditoría analizada, fortaleciendo los conocimientos técnicos, así como las habilidades relacionales de los auditores, pueden contribuir al fortalecimiento de una cultura empresarial donde se prioricen la ética empresarial, las prácticas de trasparencia informativa, y en definitiva, la calidad de la información económico-financiera suministrada a los usuarios y sometida al proceso de auditoría. Estas conclusiones se pretenden hacer extensivas a otras empresas de auditoría en futuras investigaciones.

\section{Referencias}

ABDOLMOHAMMADI, M.J.; SHANTEAU, J. (1992). Personal attributes of expert auditors. Organizational Behavior and human decision processes, 53(2): 158-172. http://dx.doi.org/10.1016/0749-5978(92)90060-K

BALSAM, S.; KRISHNAN, J.; YANG, J. (2003). Auditor industry specialization and earnings quality. Auditing: A Journal of practice and theory, 22(2): 71-97.

BONNER, S.; LEWIS, B. (1990). Determinants of auditor expertise. Journal of Accounting Research, 28: 1-20. http://dx.doi.org/10.2307/2491243

BONTIS, N.; FITZ-ENZ, J. (2002). Intellectual capital ROI: a causal map of human capital antecedents and consequents. Journal of Intellectual Capital, 3(3): 223-47. http://dx.doi.org/10.1108/14691930210435589

BROWN, H.; WRIGHT, A. (2008). Negotiation research in auditing. Accounting Horizons, 22(1): 91-109. http://dx.doi.org/10.2308/acch.2008.22.1.91

CAÑIBANO, L.; SÁNCHEZ, P.; GARCÍA, M.; CHAMINADE, C. (2002). Guidelines for managing and reporting on intangibles. Intelectual Capital Report. Fundación Airtel Móvil, Madrid. 
CAPPELLI, P.; SINGH, H. (1992). Integrating Strategic Human Resources and Strategic Management. Research Frontiers in Industrial Relations and Human Resources (pages 165-192). Industrial Relations Research Association, Madison.

CARSON, E.; RANZIJN, R.; WINEFIELD, A.; MARSDEN, H. (2004). Intellectual Capital. Journal of Intellectual Capital, 5(3): 443-463. http://dx.doi.org/10.1108/14691930410550390

CHEN, J.C.; SILVERTHORNE, C. (2008). The impact of locus of control on job stress, job performance and job satisfaction in Taiwan. Leadership and Organization Development Journal, 29(7): 572-582. http://dx.doi.org/10.1108/01437730810906326

CRASWELL, A.; FRANCIS, J.; TAYLOR, S. (1995). Auditor Brand name reputations and industry specializations. Journal of Accounting and Economics, 20: 297-322. http://dx.doi.org/10.1016/0165-4101(95)00403-3

DECHOW, P.; GE, W.; LARSON, C.R.; SLOLAN, R. (2011). Predicting material accounting misstatements. Contemporary accounting research, 28(1): 17-82. http://dx.doi.org/10.1111/j.1911-3846.2010.01041.x

DELERY, J.E.; SHAW, J.D. (2001). The strategic management of people in work organizations: Review, synthesis, and extension. Trabajo presentado en el Academy of Management Meeting, Washington.

DUNN, K.; MAYHEW, B. (2004). Audit firm industry specialization and client disclosure quality. Review of Accounting Studies, 9: 35-58. http://dx.doi.org/10.1023/B:RAST.0000013628.49401.69

FITZ-END, J. (2000). The ROI of Human Capital. New York, NY.: Amacom.

FRANCIS, J. (2011). A framework for understanding and researching audit quality. Auditing: A journal of practice \& theory, 30(2): 125-152.

HUDSON, W. (1993). Intellectual Capital: How to build it, enhance it, use it. En Jonh Wiley \& Sons, New York, NY. Citado en Bontis, N.; Fitx-end, J. (2002) (page 225).

HURTT, R. (2010). Development of a scale to measure professional skepticism. Auditing: A Journal of Practice \& Theory, 29(1): 149-171.

HYATT, T.; PRAWITT, D. (2001). Does congruence between audit structure and auditors'locus of control affect job performance?. The Accounting Review, 76: 263-274. http://dx.doi.org/10.2308/accr.2001.76.2.263

JENSEN, M.; MECKLING, W. (1976). A theory of the firm: Governance, residual claims and organizative forms. Journal of Financial Economics, 3(4): 305-360. http://dx.doi.org/10.1016/0304-405X(76)90026-X

JENSEN, K.; PAYNE, J. (2005). Audit procurement: managing audit quality and audit fees in response to agency costs. Auditing: A journal of practice \& theory, 24: 27-48. 
JOHNSON, V.; KHURANA, I.; REYNOLDS, J. (2002). Aduit firm tenure and the quality of financial reports. Contemporary accounting research, 19(4): 637-660. http://dx.doi.org/10.1506/LLTH-JXQV-8CEW-8MXD

JUDGE, T.; BONO, E. (2001). Relationship of core self-evaluations traits-self-esteem, generalized self-efficacy, locus of control, and emotional stability with job satisfaction and job performance: A meta analysis. The Journal of Applied Psychology, 86: 80-92. http://dx.doi.org/10.1037/0021-9010.86.1.80

KALECHSTEIN, A.; NOWICKI, S. (1997). A meta-analytic examination of the relationship between control expectancies and academic achievement: An 11-year follow-up to Findley and Cooper. Genetic, Social and General Psychology Monographs, 123: 29-56.

LENNOX, C.; CARAMANIS, C. (2008). Audit effort and earnings management. Journal of accounting and economics, 45(1): 116-138. http://dx.doi.org/10.1016/j.jacceco.2007.05.002

LIBBY, R. (1993). Determinants of judgment performance in accounting settings: Ability, knowledge, motivation and environment. Accounting, Organizations and Societe, 18: 425450. http://dx.doi.org/10.1016/0361-3682(93)90040-D

LIM, C.; TAN, H. (2008). Non audit service fees and audit quality: The impact of auditor specialization. Journal of accounting research, 46(1): 199-246. http://dx.doi.org/10.1111/j.1475679x.2007.00266.x

MC KNIGHT, C.; WRIGHT, W. (2011). Characteristics of relatively high performance auditors. Auditing: A Journal of practice \& theory, 30(1): 191-206.

MORONEY, R.; CAREY, P. (2011). Industry versus task based experience and auditor performance. Auditing: A journal of practice \& theory, 30(2): 1-18.

NG, T. et al. (2006). Locus of control at work: A meta-analysis. Journal of Organizational Behavior, 27: 1057-1087. http://dx.doi.org/10.1002/job.416

STEWART, T. (1997). Intellectual capital: The new wealth of organizations. New York, NY.: Doubleday. Citado en Carson, E. et al. (2004) (page 447).

SULLIVAN, P. (1998). Profiting from intellectual capital: Extracting value from innovation. En John Wiley \& Sons. New York, NY. Citado en Green, A.; Ryan, J. (2005) (page 44).

TAN, H.T. et al. (2002). The effect of task complexity on auditors' performance: The impact of accountability and knowledge. Auditing: A Journal of Practice \& Theory, 21: 81-95.

TAN, H.T. (1999). Organizational leves and perceived importance of attributes for superior audit performance. Abacus, 35(1): 77-90. http://dx.doi.org/10.1111/1467-6281.00035 
TSENG, C.Y.; JAMES, Y.J. (2005). Intellectual Capital and Corporate Value in an Emerging Economy: Empirical Study of Taiwanese Manufacturers. $R \& D$ Management, 35(2): 187-200. http://dx.doi.org/10.1111/j.1467-9310.2005.00382.x

TUBBS, R. (1992). The Effect of Experience on the Auditor's Organization and Amount of Knowledge. The Accounting Review, 67(4): 783-801.

WILLOUGHBY, M. et al. (2012). The effects of the provision of consulting services on audit reporting quality. Service Industries Journal, 32(3): 411-429. http://dx.doi.org/10.1080/02642069.2011.567415

YOUNDT, M.A.; SNELL, S.A. (2004). Human Resource Configurations, Intellectual Capital and Organizational Performance. Journal of Management Issues, 16(3): 337-360.

Intangible Capital, 2013 (www.intangiblecapital.org)

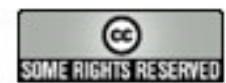

El artículo está con Reconocimiento-NoComercial 3.0 de Creative Commons. Puede copiarlo, distribuirlo y comunicarlo públicamente siempre que cite a su autor y a Intangible Capital. No lo utilice para fines comerciales. La licencia completa se puede consultar en http://creativecommons.org/licenses/by-nc/3.0/es/ 